\title{
Preliminary Design and Optimization of New Methods of Mass Clamped-free Liquid Rocket Engine Turbopump Feed System Open Cycle using the Gradient Algorithm
}

\author{
Mohammad Mohammadi \\ Malek Ashtar University of \\ Technology \\ Tehran, Iran
}

\author{
Mohammad Mehdi \\ Heydari \\ Malek Ashtar University of \\ Technology \\ Tehran, Iran
}

\author{
Sayed Hosain Ramzani \\ Malek Ashtar University of \\ Technology \\ Tehran, Iran
}

\begin{abstract}
In this paper, a new method and modern for Preliminary design and optimization mass turbopump in a liquid propellant rocket engine (LPRE) using the gradient algorithm has been developed. The cycle type of LPRE is open cycle and configuration of turbopump feed system consists of a impulse Turbine at the end of the shaft, an oxidizing centrifugal pumps and a fuel pump centrifugal that have been each other back-to-back on a common shaft. The proposed approach is based on four characteristics, optimal power system performs turbopump. These four characteristics are to achieve the desired characteristic of cavitation in pumps, in particular pump-balanced oxidizer turbine production capacity and total power consumption of the rotor pump-structures and also to estimate the mass of the turbopump feeding system as a result of this process. The design of the input parameters for the preliminary design of each component is determined and then use algorithms that are designed for the pump and turbine box design, preliminary design done. This is the optimal point, the gradient algorithm is obtained and the results with data from a real engine with a similar orbit, have been compared. Compare the results with similar inputs real engine design, the proposed method proves correct and improve the performance characteristics of the power system shows turbopump.
\end{abstract}

\section{Keywords}

Liquid Rocket engine, Turbopump, Preliminary design, feed system, Gradient algorithm

\section{INTRODUCTION}

Inducer the negative effects of pressure, corrosion, mechanical and fluid input to the turbopump reduces instability. Inducer duty increases as well as increased operating pressure for impeller inlet head enough to prevent cavitation is later. As a result, it can be, angular velocity (speed) and power-to-weight ratio increased, it raised the energetic characteristics and crime reduced turbopump set. By reducing the mass and turbopump engine, the expanded satellite cargo. However, the optimal design of power systems to find the optimal mass and rotational speed by four approaches: (1) achieve a desirable characteristic of cavitation in pumps, 2. production and consumption balance of power in the engine, turbopump power system (3) and (4) structural mass The rotor should be used. This comprehensive approach because of, the system was adopted. In this paper, using algorithms box is drawn, the initial design and then with a gradient algorithm for optimization of mass, power turbopump system, such that each expression be respected and yet objective approach to planning, which maximize the payload (cargo) is satisfied done. Extensive research on liquid fuel engine turbopump feeding system and the current achievements of the past decades is the result of efforts. Because of the extent of this study was to determine the maximum limit it to 15 years and only objective of the optimization system in history have been presented. V.N. Kurshev in 2001 on the basis of mathematical methods (minor changes) and linear mathematical equations governing the turbopump, a way to minimize the value of the maximum inlet pressure to the pump, provided that it is based on an optimization is done on the turbopump system [4]. Fatuev E.U. In 2003, a method for optimizing the structure of the RD-120 engine provides the Soyuz launch vehicle payload increase of 12.5 percent. His view only improves the performance of individual subsystems of the engine [5]. Dr. David Japikse and K. Fetfatsidi, in 2003, the basic design of a fuel and oxidizer turbopump shaft's single. In this design, all the way up the fuel pump and oxidizer turbopump aim was to design (the initial design optimization) is common. Different trims on the turbine blades and different machined blades on the pump impeller and diffusers allow adaptation to the specific oxidizer and fuel pumping requirements. The initial design was acceptable amount of size, weight, cost and performance provided Dynamics- fluids [6].

Y. Demyanenko in 2006 to study single-axle turbopump engine's fuel. In this study, the engines RD-120, SSME and the RD-0146 were reviewed and analyzed. In this paper, improving dynamic performance rotor, which includes attenuation elastic support rotor and rotor balance in performance speed range is developed and offered. Use of a booster pump oxidizer turbopump uniaxial new possibilities for the use of log RL10 RL-10 so successful is its structure. Single turbopump-shaft engine thrust set to rise to $40 \%$ and reducing the vapor pressure at the pump inlet pressure pump hydrogen and oxygen [7]. Michael A. Arguello in 2008 in his doctoral dissertation on conceptual design of liquid hydrogen turbopump pay. Intended to improve motor function in the final phase of the motor DEAN, split-flow design to reduce power requirements at the second stage. Performance parameters are required for the design of output pressure, increasing the pump head, output stage and power. This will be turbine and power requirements by $52 \%$ compared to the full flow decrease. As a result, fluctuations in the level and reduced the effective length turbine blade, rotor life will be better and geometric effects [8]. MIZUNO Tsutomu and KOBAYASHI Satos, in 2010, LE-X's advanced design 
turbopump engine. The new cryogenic engine booster engine performance, high reliability and low cost, so that the next generations of engines thrower Japanese carrier is used [9]. William L Murray and Matthew W. Steiner, in a 2014 article on the design and analysis of high-speed, high-pressure turbopump kerosene / hydrogen peroxide was used. They study at Purdue University found that using the silver catalyst bed to decompose hydrogen peroxide can have a better performance in loading and higher pressure for the propulsion imagine. They showed that utilizes modern technology to analyze the factors driving at high pressure and kerosene significantly enhance the performance of a turbopump engine (single shaft, radial turbine, centrifugal pump) and lighter weight reduction rocket engine weight to 5,000 pounds. The turbopump is extremely small and has a high speed [10].

\section{THE PRELIMINARY DESIGN PROCESS}

The governing equations for the Preliminary design of the main components of the turbopump system (pump fuel oxidizer and impulse turbine) is expressed. Liquid rocket engine discussed in this article open cycle turbopump engine is a type of cryogenic propellant $\left(L_{\mathrm{O}_{2}}, R P-1\right)$ is. The main turbopump engine includes a gas turbine of the type of single-stage impulse-rich fluid oxidizing agent, a centrifugal pump oxidizer and a centrifugal pump fuel. A summary of the process of designing the algorithm flowchart in Figure 1 shows the Preliminary design box. At this step having input parameters such as mass flow rate through the pumps, pressure at the outlet of inertia pumps, pressure at rest at the entrance pumps, fluid density, viscosity, vapor pressure and kinematic parameters such as volumetric flow rate of liquid propellant components can be head required to pump the oxidizer and fuel pumps, centrifugal calculated. In this step as a parameter outlet pressure constant and independent of the other parameters for the output pressure suitable for pumping fuel and oxidant to the fuel injector can be considered.

\subsection{Determine the angular velocity and special rate}

It should be noted that to ensure constant air pressure is always greater than the pressure required to design. So the $N P S H_{\text {ind }}$ according to the Net Positive Suction Reserve Head for the pump is considered Reserve, is calculated. At first need to determine the angular velocity of the pump to a maximum value of specific speed pump cavitation $C_{\text {CPB, max }}$ determined to prevent cavitation. Range is $\left(3000 \leq C_{\mathrm{CPB}, \max } \leq 4500\right)$ [14]. Having $C_{\mathrm{CPB}, \max }$ value using equation (1) the angular velocity of the pump to be achieved:

$$
\omega=C_{\mathrm{CPB}, \max } \cdot N P S H_{\text {ind }}{ }^{0.75} /(298 \sqrt{V})
$$

For calculating the diameter of the Bush factor $k_{d s}$ need an initial amount of data to be pumped to the calculations done in the next step. Initial guess for power stations in the input parameters were taken into account during the calculation algorithm is correct. This factor depends on the shaft allowable torsional stress. And according to material in $\left(\mathrm{N} / \mathrm{m}^{2}\right)$ shaft between the $\left(10^{8} \leq \tau_{\text {allaw }} \leq 2 * 10^{8}\right)$ considered. At this stage should be noted that Bush factor diameter ratio is calculated within the specified range. In the opposite, according to the flowchart, with regard to guess the right of the power station, the calculation begins again.

\subsection{Calculation of inducer and centrifugal wheel input}

Among the important parameters that are calculated in this step include, inducer Bush diameter, outer diameter inducer, shaft pump diameter, And the peripheral speed in diameter are inducer. By measuring the $\operatorname{amount}\left(k_{D s}\right)$, angle and volumetric flow rate can be calculated outside diameter inducer. Multiplied by a factor of Bush diameter inducer in outer diameter inducer, the diameter of inducer Bush also calculated. Having Bush diameter inducer Pump shaft diameter $\left(d_{s h}\right)$ can be calculated. The next step is to calculate the mean diameter and the angular velocity and volumetric flow rate can be calculated Peripheral speed in inducer diameter (Mean diameter inducer is equal to average, Bush diameter and outer diameter inducer). The design assumes outer diameter inducer is equal to the diameter of the inlet centrifugal wheel. Having a mean diameter inducer can mean diameter inlet centrifugal wheel to be calculated. The diameter ratio of input to output can be impeller for the first approximation, be calculated as follows:

$$
\frac{D_{1}}{D_{2}}=0.007 \frac{\varphi_{D} \cdot k_{D_{0}}}{\sqrt[3]{i}}\left(\sqrt{k_{z} \cdot \eta_{\Gamma} \cdot\left(1-q_{p}\right)}\right) \cdot n_{s}^{2 / 3}
$$

In the above equation to solve this problem, it is assumed $q_{p}=0.15$ and $k_{z} \cdot \eta_{\Gamma}=0.66$ that by repeating the solution, the amount given is correct [11]. By calculating the diameter wheels and an average diameter wheel input, the width of the input wheel is calculated. It should be noted that the design always $\frac{D_{1}}{D_{2}} \leq 0.6$ and $k_{D_{0}} \leq 7.2$ is established. Otherwise, according to the flowchart in Figure 1 is expressed, should be optimized coefficient inducer diameter $\left(k_{D s}\right)$ is corrected. To calculate the induction step, need to angle the output current and the average diameter induced peripheral speed ratio (eq.3) induction to calculate the output:

$$
\bar{C}_{2 u c p}=\left[\left(0.5\left\{\lambda_{c p b}\left[\left(1-\bar{C}_{2 u c p}\right)^{2}+\left(\frac{c_{1 z}}{u_{c p}}\right)^{2}\right]+\bar{C}_{2 u c p}{ }^{2}+\left(\frac{c_{1 z}}{u_{c p}}\right)\right\}\right)+\frac{\Delta H_{c p}}{u_{c p}}-\frac{N P S H}{u_{p p}}\right] /
$$

By solving a nonlinear equation, $(\bar{C} 2 u c p)$ is obtained. It should be noted that this equation has three questions regarding the extent to which the design for which it is intended $\left(0.2 \leq \bar{C}_{2 u c p} \leq 0.5\right)$, will always be a fundamental solution [11]. With calculation $\left(\bar{C}_{2 u c p}\right)$, at the beginning of the output flow angle inducer mean diameter and then the step of inducer is calculate.

\subsection{Calculation Of Cavitation}

For calculation Special speed pump cavitation, the first need is to be calculated correction factor $\left(a_{0}\right)$. In the next step, cavitation factor inducer and Lateral loss coefficient are calculated. However, by calculating the above parameters, $N P S H_{\text {ind }}$ can be used to more accurately calculate the following equation: 
$\operatorname{NPSH}_{i n d}=\left[\left(\frac{c_{1 z}}{u_{c p}}\right)^{2}\left(1+\xi_{f t}\right)+\lambda_{\text {lcpp }}\left(1+\left(\frac{c_{1 z}}{u_{c p}}\right)^{2}\right)\right] *$ $*\left(\frac{\boldsymbol{u}_{c p}^{2}}{2}\right)$

By calculating $N P S H_{\text {ind }}$, can be calculated Special speed pump cavitation $\left(C_{C P B}\right)$ :

$$
C_{C P B}=298\left(\frac{\omega \sqrt{W}}{N P S H_{\text {ind }}^{3 / 4}}\right)
$$

It should be noted that cavitation occurs in the pump system, the maximum Special speed cavitation designated higher than Special speed cavitation $\left(C_{\mathrm{CPB} \text {, } \max } \geq C_{C P B}\right)$ that of equation (5) is obtained [11]. Or in other words the difference between the two special speeds must be less than $5 \%$ is the cavitation does not occur in the pump. Otherwise, according to the flowchart in Figure 1, have a maximum Special speed cavitation corrected and the design of the block to be started to determine the angular velocity. In the case of indicating the desired contact, designed to continue to the next step which is described below, explains.

\subsection{Calculation of geometric dimensions and thermodynamic parameters impeller}

At this stage, the rake angle and blade angle at the entrance wheel is calculated. It should be noted that the blade angle at the entrance wheel is between $\left(15^{\circ} \leq \beta_{1 b} \leq 30^{\circ}\right)$ [11]. Whit calculation the coefficient head, we can calculate the Wheel peripheral speed and output diameter of the wheel. One of the things that should be noted in this section, calculate $D_{2}$ and $\bar{D}_{1}=\frac{D_{1}}{D_{2}}$ range that was mentioned in the previous section.

Blade output width to calculate, first we need to determine the angle of the blade on the output wheel and then output width to be determined. It should be noted that the Blade angle wheel output is between $\left(15^{\circ} \leq \beta_{2 b} \leq 20^{\circ}\right)$ [11]. However, it should be noted that the amount $\beta_{2 b}<\beta_{1 b}$. Otherwise, the first step is to continue the process to solve the design (input data) will return. And by correcting the minimum input inertia pressure, and solution design process is resumed. $\beta_{2 b}$ Parameter is calculated according to the number of wheel blades.

\subsection{Design diffuser and volute}

Fluid energy output of the wheel, is in the form of kinetic and potential. The pressure drop in the ducts, pumps, proportional to the square of speed. Thus, the speed of the fluid at the entrance to the volute, lower the pump efficiency will be improved. In fact, the diffuser channel is residing with or without fins. That is between the wheel and volute. And by increasing the flow rate decreases and pressure increases.

\subsubsection{Calculation of geometry diffuser with fins}

According to Figure (2) fined diffuser, if not much space between the fins, each fin can be made in the form of a circular arc, assuming that the width $b_{2}$ considered as a throat diffuser. Along the curve of fin shall be such that the angle between the fin channel disruption to the width $b_{3}$ Not more than 8 to $12 \mathrm{~mm}$ [11].

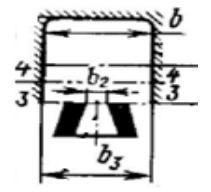

Fig 2. A schematic of the diffuser centrifugal pump [11].

\subsubsection{The calculation of volute geometry}

Mansour collect the fluid at the outlet of the wheel or diffuser in centrifugal pumps, volute is usually used. This type of volute surface area of the nose and throat usually proportional to the increase in the flow rate of the wheel, increases. Increased cross-sectional area in the direction of rotation of the wheel. The volute design is necessary following characteristics to be determined; General shaped cross-section volute, the main diameter $\left(D_{3}\right)$, Input width volute or the diameter equivalent of throat $\left(d_{e t}\right)$, Cape of angle volute $\left(\alpha_{4}\right)$.

\subsection{Calculation Of Efficiency, Power Consumption And Losses At The Pump}

\subsubsection{Calculation Loss coefficients}

According to an equivalent of angle diffuser $\left(\alpha_{3}\right)$, can loss coefficient of the diffuser and calculate the loss rate in output diffuser. But it should be noted that the values listed to be in the range of $\left(0.15 \leq l_{f d} \leq 0.35\right) \&\left(0.15 \leq l_{f c} \leq 0.3\right)$.

2.6.2 Calculation of leakage in the pump wheel Non-contact seals static shoulder, are in widespread use in turbopumps. Gap seal small $\left(\delta_{y}\right)$, Fluid friction during the gap $\left(l_{y}\right)$, Lack of fluid leakage. Degree of development of this type seals, by determined the flow rate coefficient $(\mu)$.

With increased sealing capability, $(\mu)$-value decreases, and this means increased hydraulic resistance is gap [14]. The discharge coefficient seal wheel and length gap can be calculated. The above parameters can be calculated as volumetric flow rate of leakage in the front of the seal and the total volumetric flow leakage through the gap to calculate the wheel.

\subsubsection{Calculation the coefficient of friction and the power friction wheel}

By calculating the total leakage volumetric flow rate of the wheel, it can calculate pump efficiency. Assuming that the leakage rate is equal on both sides of the pump, pump efficiency is equal to:

$$
\eta_{p}=\frac{1}{\left(1+1,33 \cdot 10^{6} \mu \frac{\delta_{y}}{D_{y}}\left(\frac{D_{y}}{D_{1}}\right)^{2}\left(\frac{D_{1}}{D_{2}}\right)^{2} \frac{A}{\left[\eta_{z} k_{z}\left(1-q_{p}\right)\right]^{3 / 2} n_{s}^{2}}\right)}
$$




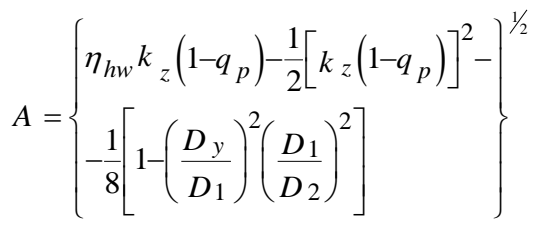

Using the parameters calculated in the previous section, It can be said that the efficiency of the pump wheel:

$$
\begin{aligned}
\eta_{p d}= & \frac{C_{f d}}{C_{f d}+1,3 \cdot 10^{-5}\left(\eta_{h p}\right)_{\varphi \neq 0}{ }^{3 / 2}\left[k_{z}\left(1-q_{p}\right)\right]^{5 / 2} n_{s}^{2}+17,2 \mu \times} \\
& \times \frac{\delta_{y}}{D_{y}}\left(\frac{D_{y}}{D_{1}}\right)^{2}\left(\frac{D_{1}}{D_{2}}\right)^{2}\left[k_{z}\left(1-q_{p}\right)\right] A
\end{aligned}
$$

Internal power pump efficiency can be expressed as follows

$$
\eta_{p i}=\eta_{p d} \cdot\left(\eta_{h p}\right)_{\varphi \neq 0} \eta_{p}
$$

In the absence of pump impeller seals, mechanical efficiency $\left(\eta_{m}\right)$ is between 0.995 to 0.99 , but if you use the pump impeller seals, mechanical efficiency is reduced and between 0.995 to 0.97 . This data can be used in the initial calculations of mechanical efficiency [14]. According to the design the pumps of this type of seal is used mechanical efficiency can be considered approximately equal to 0.97 . According to the parameters that the overall efficiency of the pump:

$$
\eta_{T P}=\eta_{p i}+\eta_{m}
$$

Pump system by parameters rocket engine power, flow, pressure and efficiency of the pump is determined. Power requirement efficiency pumps, volumetric flow rate and head required depend on:

$$
N_{P}=\rho \dot{V} H / \eta_{T P}
$$

\section{PRELIMINARY DESIGN OF SINGLE-STAGE IMPULSE TURBINE} In this paper, using the design code was developed by writers, single-stage turbine is designed. Reference radius calculations, middle radius. The code, after receiving input profile aerotrmodynamic turbines, other parameters and specifications required for the blade profiles, geometry nozzles and turbine wheel, losses and productivity measures. A summary of the design process flowchart algorithm designed box first single-stage impulse turbine (Fig.3) is shown.

\subsection{Input parameters}

The input data includes angular velocity of the shaft, Power requirement pump, turbine inlet temperature of inactivity, input pressure and gas properties.

\subsection{Parameters of the gas flow}

The estimated flow profile in the preliminary design is usually done in the mid-height blade. Care must be taken that step, pump output such as; Power requirement pump or angular velocity of the shaft and the output parameters such as pressure and temperature of the hot gas from the gas generator, with a small drop which is negligible, as the turbine inlet are considered. In this step with a geometric expansion ratio and the peripheral speed average diameter of the turbine, The calculation of the mean diameter, adiabatic work, adiabatic speed, special speed turbines done[11]. Then, knowing the turbine inlet flow rate or the output of the gas generator $\left(m_{T}\right)$, Special turbine speed can be calculated as follows:

$$
n_{s t}=\frac{193.3 \omega \sqrt{m_{T} / \rho}}{L_{a d}{ }^{0.75}}
$$

At this stage, care must be taken that the $\left(u / C_{a d}>0.2\right)$ to be, otherwise you have to input speed hot gas in the turbine according to box algorithm (Fig.3) is correct.

\subsection{The Estimated Gas Flow Parameters \\ Between A Nozzle And Turbine Wheel}

After calculating the average diameter of the turbine wheel and adiabatic speed calculations necessary to achieve critical velocity of sound, pressure coefficient input, static temperature, static temperature at the entrance to the turbine wheel And Mach number at the start of the relative speed. Therefore respectively, relative speed and stasis temperature at the nozzle outlet can be calculated as follows:

$$
\begin{aligned}
& \omega_{1}=\sqrt{\left(C_{1} \operatorname{Cos} \alpha_{1}-u\right)^{2}+\left(C_{1} \sin \alpha_{1}\right)^{2}} \\
& T_{{ }_{0_{C_{1}}}}=T_{1, a d}+\left(\frac{k-1}{2 k R}\left(C_{1}\right)^{2}\right)
\end{aligned}
$$

\subsection{Estimated blade height of the turbine wheel}

By selecting the initial value for partial acceptance factor $(\varepsilon)$ can be calculated blade height and relative height blade turbine wheel turbine wheel. But it should be noted that the range of $\left(0.2 \leq \frac{h_{1}}{D_{c p}} \leq 0.5\right)$ is always true. If you have an initial value of partial acceptance factor corrected. Partial acceptance rate is generally considered to be 0.31 times the first estimate. Blade height of the turbine wheel is calculated as follows [11]:

$$
h_{1}=\frac{m_{T}}{\pi \cdot D_{c p} \cdot \rho_{1} \cdot \varphi \cdot \operatorname{Sin}\left(\alpha_{1}\right) \cdot \varepsilon}
$$

\subsection{Estimated tapered nozzle geometry}

By selecting the initial value for the distance between the blade and nozzle tip $\left(\Delta h_{p}=0.0005 \mathrm{~m}\right)$ and Distance between the blade and nozzle $\operatorname{root}\left(\Delta h_{\text {bosh }}=0.0005 \mathrm{~m}\right)$, Can be used the first approximation, nozzle blade height and Minimum level of nozzles can be calculated. In the initial selection the distance should be the range of $\left(1 \mathrm{~mm} \leq \Delta h_{p} \leq 2 \mathrm{~mm}\right)$, $\left(0 \leq \Delta h_{\text {bosh }} \leq 1 \mathrm{~mm}\right)$ note. According to the subjects above, it can area of the tapered end of the nozzle, area of the nozzle outlet, Number of nozzles, Step nozzle and minimum diameter of nozzle (Eq.16) is achieved.

$$
d_{\min }=\sqrt{4 F_{\min } / \pi Z_{c}}
$$


In this step, assuming equivalent Blade wheel height the turbine inlet and outlet, has been $\left(0.3 \leq \frac{d_{\min }}{h_{2}} \leq 0.6\right)$ [14]. Otherwise, as shown in Figure 2 should be corrected values of $\Delta h_{p}$ and $\Delta h_{\text {bosh }}$ in problem solving.

\subsection{Gas Flow Parameters In The Turbine Wheel}

Aerothermodynamics calculations turbine wheel channels, this type of turbine flow with partial acceptance, will be carried. The first parameter is the angle of the turbine wheel inlet. According to experimental data for this type of turbines and according to the type of application, from the values in the reference 11; Relative pitch blades, angle Blade, Blade width is considered by default, Using the values obtained with respect to the assumptions, The respectively blade chord and their numbers (Eq.17) is calculated.

$$
Z=\frac{\pi b_{c} D_{c p}}{\bar{t}}
$$

This code, loss values Such as loss profiles, secondary flow, frictional loss blades and bandages, loss associated with the partial acceptance and with high accuracy by using the waste model "Avsianikov.B" measures [11]. Studies show that the turbine efficiency is strongly dependent on the percentage of partial acceptance. At this stage should be noted that the range of $\left(0.3 \leq \frac{b}{D_{c p}} \leq 0.5\right)$ is always on. Otherwise, to calculate the correct number of blades, you must be a correct blade width.

Since the, height blade in output and input are equal $\left(h_{2}=h_{1}\right)$ , the angle is calculated at the output of the turbine wheel.

\subsection{Estimates of work, power and turbine efficiency}

Finally, should the flow parameters of the turbine disc as; Environmental efficiency, Special of work environment, Friction power turbine wheel, Friction power bandaged, Power dissipated, Power effective(eq.18) and Effective efficiency turbines(eq.19) are calculated. In this section you should note that the range of $\left(0.017 \leq \frac{2 . \Delta_{r}}{D_{\text {band }}} \leq 0.21\right)$ is always true. Otherwise the value $\Delta_{r}$ (minimum radial distance of bandages and body) is correct. The design is generally in the range of $1.5 \mathrm{~mm}$ and $\left(1 \mathrm{~mm} \leq \Delta_{r} \leq 3 \mathrm{~mm}\right)$ is defined.

$$
\begin{aligned}
& N_{T e}=\left[\left(m_{T}-m_{y}\right) L_{u}\right]-N_{d f}-N_{L}-N_{b} \\
& \eta_{T e}=L_{T} / L_{a d}
\end{aligned}
$$

\section{THE BASIC THEORY AND PRINCIPLES OF PRELIMINARY DESIGN AND OPTIMIZE FEEDING SYSTEM}

According to the four approaches mentioned in the introduction to it, Basic theory and preliminary design principles and Optimization engine liquid fuel feeding system according to the output parameters obtained from the previous section, the following four groups studied:

\subsection{Estimating the mass of turbopump feeding system}

Generally estimate the mass liquid propellant feeding system is a statistical perspective. Statistical research shows[12], $[13],[14] \&[15]$ that factors affecting turbopump mass, including; rotor angular velocity or shaft, Head Pumps, the rate of flow through the pumps and Power required by pumps. Thus, the factor according to the following equation [15], it has been seen that the effect of these four parameters as follows:

$$
m_{t p}=A\left(\frac{N_{P, \text { oxid }}+N_{P, \text { fule }}}{\omega}\right)^{B}
$$

The most decisive factor in the above equation, is turbopump rotor angular velocity. In other sources, relations with different perspectives (e.g. with regard to parameters such as; turbine production power and number stages of turbines) is provided [18], It can also be used but is not limited to providing them the opportunity.

\subsection{Cavitation characteristics of the pump} and the rotor angular velocity

Developed an algorithm that could, Liquid propellant feeding system is designed to do, more than anything, is based on the oxidizer pump cavitation boundary. In other words, is

$\left(C_{\text {CPB,max }} \geq C_{C P B}\right)$. The reason should be more willing to cavitation oxidizer component (due to high vapor pressure) to be search [19]; In fact, this factor determines the angular velocity of the oxidizer pumps. However, equation (20) show the increase rotor angular velocity in the main turbopump, it will lead to a reduction in mass. That means of angular velocity can be obtained for the main turbopump in which the mass feeding system (main turbopump) is minimized.

\subsection{Balance of power}

Turbopump angular velocity affects the efficiency and power of pumps and turbines. So the algorithm box (Figure 4), must balance production power turbine and power requirement pumps, Due to the amount of fuel and oxidizer mass rate and to establish the flow rate of the components (energy balance). According to Eq. (18) and (11) the balances of the angular velocity, calculated by Eq. (21) is handled.

$$
\sum N P_{P}=\sum N_{T e}
$$

\subsection{Rotor structures}

The rotor diameter is installed in the backrest, of the power turbine (total power pumps) and the rotor angular velocity is obtained[19]:

$$
d=\sqrt[3]{\frac{5 \cdot 1_{N_{T e}}}{\omega \cdot \tau_{\text {allow }}}}
$$

The turbopump rotor is made of the amount of shear stress are used in the range of 100 to 200 (Mpa) and here is the basis of also the same amount. This suggests that one approach to optimization of turbopump rotor, the rotor is less which leads to mass - the use of materials that have more shear strength. Peripheral speed $(u)$ of the rotor in the area of support is calculated by Eq.23:

$$
u=0.5 \omega d
$$


Work experience, Liquid fuel engine turbomachinery, Show that the speed of environmental support, Number about 100 to 110 meters per second. So turbopump angular velocity must be such that it satisfies the condition.

\section{DESCRIBES THE ALGORITHM TO CALCULATE THE ANGULAR VELOCITY AND MASS OPTIMIZATION}

The logic of the proposed method to calculate the angular velocity and mass-optimized, the algorithm is a box, shown in Figure4. The algorithm is based on four preliminary design criteria for turbopump feeding system that were noted in the previous section, the engine power to the system discussed in this article are set. Algorithm for, liquid rocket engine turbopump open cycle it is written. Input data to start the algorithm; Minimum inlet pressure to inducer pump, mass rate fuel and oxidizer In determined motor orbit, The maximum inlet pressure to the turbine, gas turbine inlet minimum flow rate of the gas generator and thermodynamic characteristics of the gas turbine inlet (fixed adiabatic gas, gas constant and gas temperature) is.

\subsection{Gradient algorithm}

According to this logic, using equations and input data is expressed, In a certain value $k_{D s}$ (coefficient inducer diameter oxidizer pumps), head, pump efficiency and power pump can be calculated (Fig.1). With this data in a number of turbine inlet pressures, the power turbine is calculated and then the balance of power requirement pumps and power turbine, are controlled. In the absence of convergence with reasonable accuracy, the results with other values of input pressure are checked. Input pressure, establish the balance of power $\left(\sum N P_{P}=\sum N_{T e}\right)$, to continue the calculation used. The rotor angular velocity is considered, according to equation (22), the minimum diameter of the rotor turbopump seating the bearing is calculated. As you know, speed peripheral seating Ball bearings, is limiting type of ball bearings. With speed control environment, the data obtained from this calculations (including angular velocity, power requirement pumps power turbine, Heads and The rate of flow through the pump) calculations the mass of the turbopump is placed. Usually Eq.24 between the inducer diameter coefficient oxidizer pumps and fuel is established [17]. In fact, different values $k_{D s}$ (inducer diameter coefficient oxidizer pumps) of space for the optimal solutions are created. Process that was followed, with a lot of other $\mathrm{d}$ is repeated. The gradient algorithm is able to determine the optimal amount $k_{D s}$ its basic geometry pumps and turbopump rotor angular velocity and basic geometry turbine also be determined.

$$
k_{D s, f u}=0.9 k_{D s}
$$

\section{RESULTS}

Input problem-solving for the preliminary design of the system feeding liquid rocket engine, in Table1 liquid rocket engine bipropellant cryogenic liquid oxygen and kerosene is been proposed. The values presented in Table $1, \mathrm{~F}-1$ is the nominal values of the engine [20].
Table.1- Input parameters preliminary design and optimal

\begin{tabular}{|c|c|c|}
\hline Title & Symbol & $\begin{array}{l}\text { amount } \\
\text { (unit) }\end{array}$ \\
\hline $\begin{array}{l}\text { Minimum inlet } \\
\text { pressure oxidizer }\end{array}$ & $P_{\text {in.min.ox }}$ & $0.44(\mathrm{MPa})$ \\
\hline $\begin{array}{l}\text { Minimum inlet } \\
\text { pressure fuel }\end{array}$ & $P_{\text {in.min.fu }}$ & $0.31(\mathrm{MPa})$ \\
\hline $\begin{array}{l}\text { oxidizer mass flow } \\
\text { rate }\end{array}$ & $m_{o x}^{\square}$ & $1805(\mathrm{~kg} / \mathrm{s})$ \\
\hline fuel mass flow rate & $m_{f u}^{\square}$ & $795(\mathrm{~kg} / \mathrm{s})$ \\
\hline $\begin{array}{l}\text { turbine inlet gas } \\
\text { temperature }\end{array}$ & & $1062(k)$ \\
\hline fixed Gas & & $400(j / \mathrm{kg} . \mathrm{k})$ \\
\hline adiabatic constant & & 1.33 \\
\hline
\end{tabular}

\subsection{Results Solved Using Gradient Algorithm}

According to Figure 4 algorithm, mass change feeding system in terms Turbopump angular velocity can be calculated. The result of this calculation is shown in Fig.5 to Fig.8 and as well as for integrated is depicted in Fig. 9. Figure 5 to Figure 9, is show, mass feeding system turbopump engine in any $k_{D s}$ (inducer diameter coefficient oxidizer pumps) is a minimum. Each of these can be minimized, called local minima. And if the design approach is based on the Reciprocating Code: like what is presented in this paper is not, the designer can make a mistake in choosing the point design. Minimum Points Zinc diagrams to change $k_{D s}$ is inclined to the left and only the minimum can't be found for the various diagrams. These diagrams show that the mass of the engine feeding system, per $k_{D s}$ has a minimum point is relative to the minimum, maximum angular velocity. The concept of maximum, the turbopump angular velocity is that can be, the maximum round for turbopump achieved, that mass feeding system is the minimum. In other words was proved turbopump rotor angular velocity with the right choice of, Can be the engine feeding system and consequently, The liquid rocket engine with the lowest mass is design. It is clear that, this is one of the main goals engine for liquid rocket and satellite designer. Input for data in Table. 1 is provided, the optimal point of the feeding system and compare it with the engine sample (F-1) are shown in Table.2. Compare shows the preliminary design gradient method with real results with an error of less than half a percent difference. From view approximation preliminary design engineering, very acceptable. On the other hand the proposed method correctly is approved.

\section{SUMMARY}

The results show that, on the stage preliminary design turbopump engine liquid rocket feeding system, can be successfully gradient method expressed in the design. One of the reasons, very near match with the results sample the algorithm expressed box (Figure 4), is implicated, all design parameters and applied to all Design constraints. 
Table.2- The optimization of feeding system and compare it with sample engine

\begin{tabular}{|c|c|c|c|}
\hline Parameter & $\begin{array}{c}\text { Value } \\
\text { Optimization }\end{array}$ & $\begin{array}{c}\text { The sample } \\
\text { engine }\end{array}$ & $\begin{array}{c}\text { Error } \\
\text { rate }\end{array}$ \\
\hline$K_{D s}$ & 8 & 7.75 & $0.32 \%$ \\
\hline$\omega_{t p}$ & $576.3(\mathrm{rad} / \mathrm{s})$ & $574(\mathrm{rad} / \mathrm{s})$ & Very well \\
\hline$m_{t p}$ & $1435 \mathrm{~kg}$ & $1429 \mathrm{~kg}$ & $0.4 \%$ \\
\hline
\end{tabular}

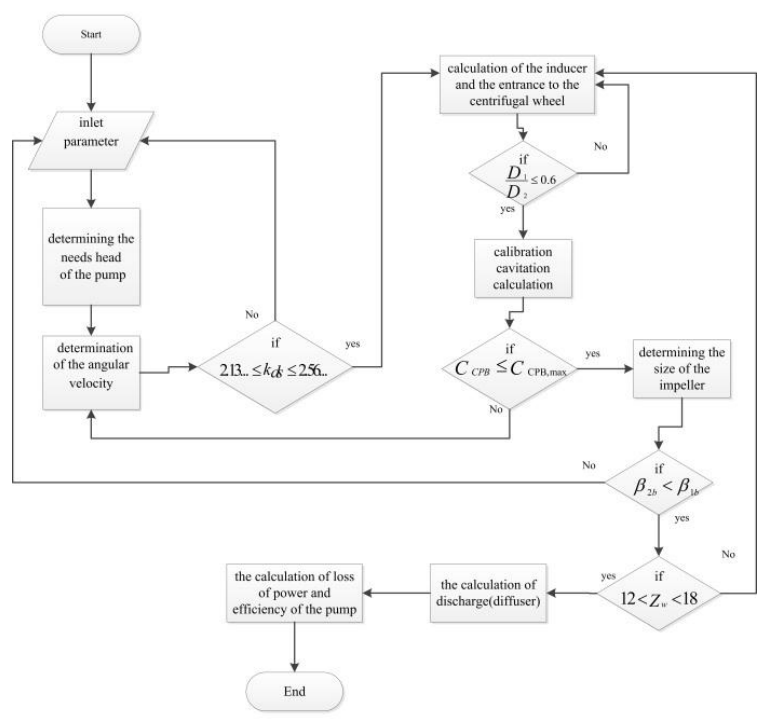

Fig 1: Box algorithm Preliminary Design centrifugal pump

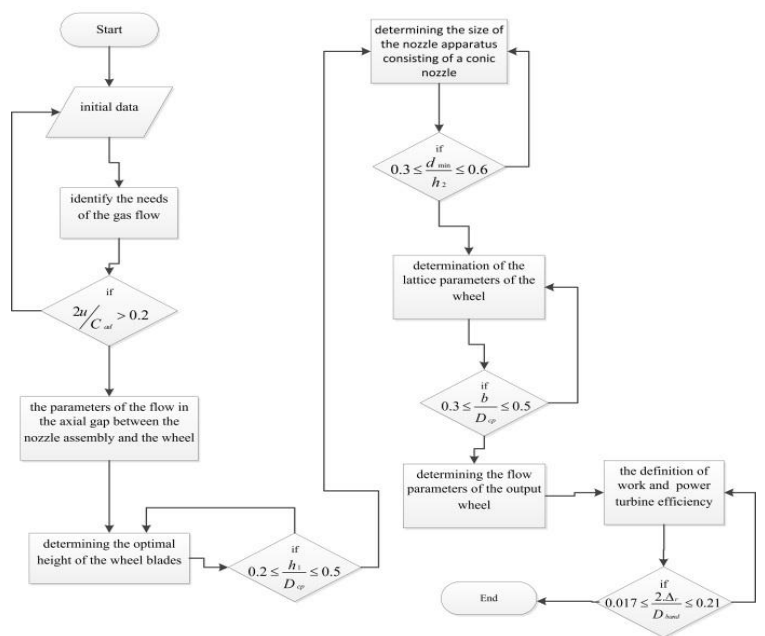

Fig3: Box algorithm Preliminary Design of single-stage impulse Turbine

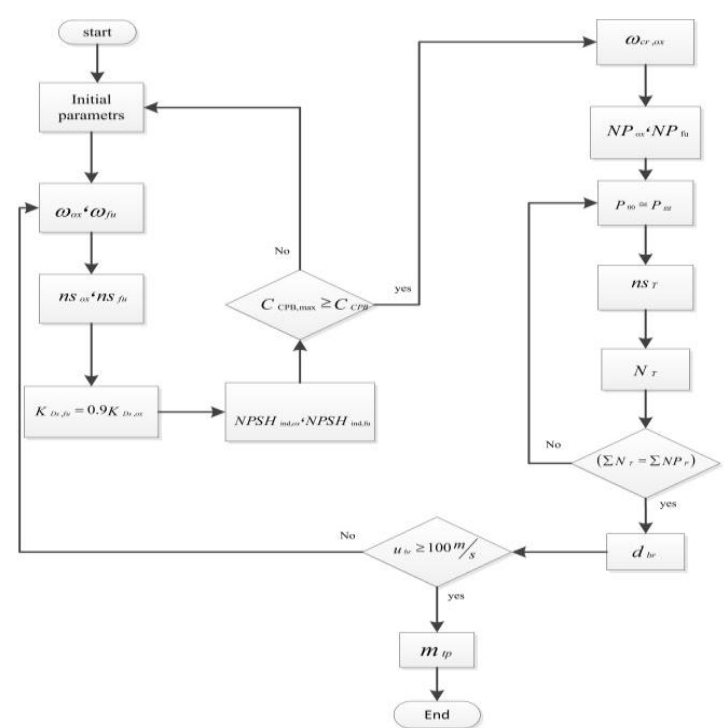

Fig4: Algorithm box calculation angular velocity and mass feeding system optimized turbopump

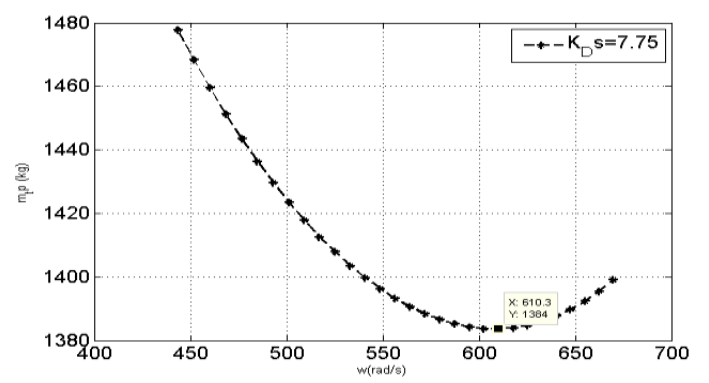

Fig5: The mass change feeding system according to the angular velocity in $K_{D s}=7.75$

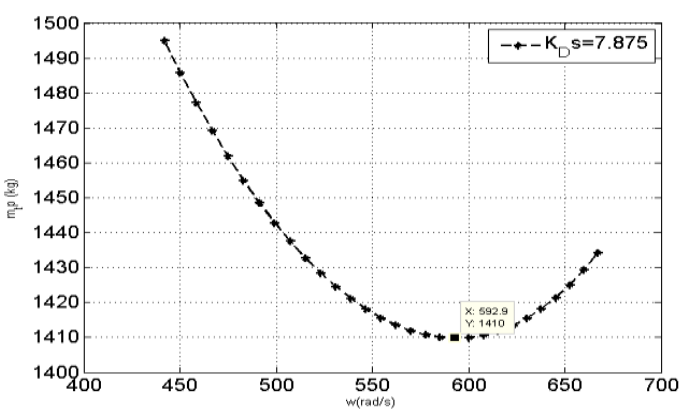

Fig6: The mass change feeding system according to the angular velocity in $K_{D s}=7.875$

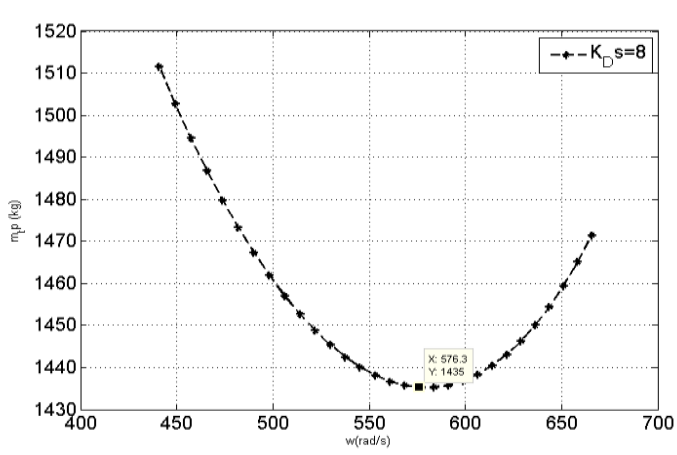

Fig7: The mass change feeding system according to the angular velocity in $K_{D s}=8$ 


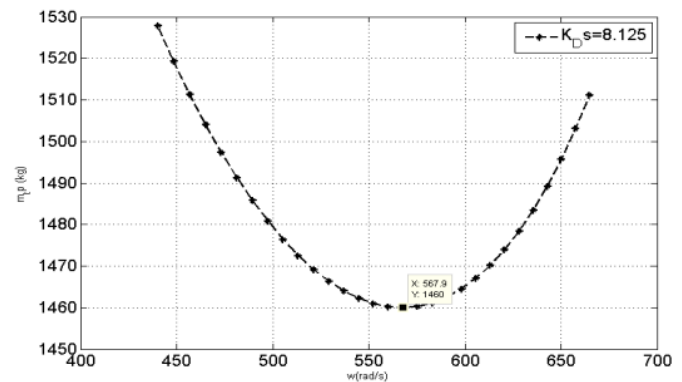

Fig8: The mass change feeding system according to the angular velocity in $K_{D s}=8.125$

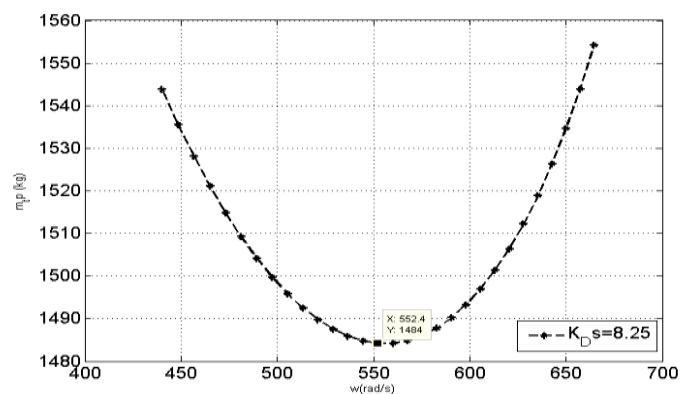

Fig9: The mass change feeding system according to the angular velocity in $K_{D s}=8.25$

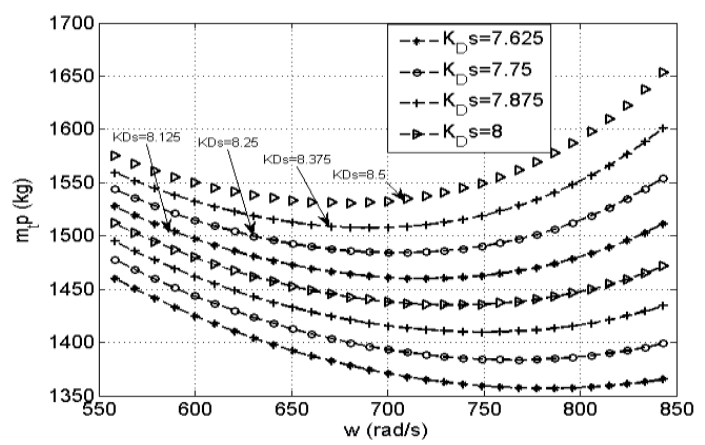

Fig10: Changes The mass feeding system aaccording to the angular velocity In various $K_{D s}$

\section{REFERENCES}

[1] F.W. Huber, PD. Johnson" Design of advanced turbopump drive turbines for National Launch System application" AIAAJSAE /AS M E JASEE 28th Joint Propulsion Conference and Exhibit, July 6-8, 1992.

[2] R.J. Gerth, Spreadsheet Approach to Minimum Cost Tolerancing For Rocket Engines,Computers industrial Engineering Vol. 27, Nos 1-4, pp.549-552,1994

[3] V.H. Katherine, J. Bailey,A. Majumdar, Nnmerical prediction of transient Axial thrust and intrrnal flows in rocket engine turbopump, AIAA-99-2189,1999

[4] V.N. Kurshev, Two problems of analyzing of turbopump of LPE, KAI, ISSN 0579-2975.Avitsionaya Tekhnika ,N2, 2001,(In Russian).
[5] E.U. Fatuev, Investigation of modificatiom of LPE RD120 for 1-st stage of Space launchvehicle,PHD tesis in MGTU, 2003, (In Russian).

[6] Dr. David Japikse \& K. Fetfatsidi" Preliminary Design of a Singular Fuel/Oxidizer Turbopump " 39th AIAA/ASME/SAE/ASEE Joint Propulsion Conference and Exhibit Von Braun Center, Huntsville, Alabama July $20-23,2003$.

[7] 1 Y. Demyanenko, A. Dmitrenko, V.Rachuk"SingleShaft Turbopumps in Liquid Rocket Engines"AIAA/ASME/SAE/ASEE Joint Propulsion Conference \& Exhibit, Sacramento, California, pp 9 12, July 2006.

[8] Michael A. Arguello"THE CONCEPT DESIGN OF A SPLIT FLOW LIQUID HYDROGEN TURBOPUMP"DEPARTMENT OF THE AIR FORCE AIR UNIVERSITY, March 2008.

[9] MIZUNO Tsutomu \& KOBAYASHI Satoshi" Development of Turbopump for LE-X Engine" P. E. Jp, Manager, Space Technology Group, Research \& Engineering Division, Aero-Engine \& Space Operations , 2010.

[10] William L. Murray, Matthew W. Steiner" Design and Analysis of a High Speed, High Pressure Peroxide/RP-1 Turbopump" Aeronautics and Astronautics, Purdue University, Indiana, AIAA, 2014

[11] Avsianikov, B., "Theory and calculation of feed systems's elements of liquid propellant rocket engines", Mashinostroyne, In Russian , 1983

[12] Avsianikov, B., "Theory and calculation of aggregates supply of liquid rocket engines" Mashinostroyne, In Russian, 1971.

[13] A.F.Goref, D.N.Surnov, D.D.Sevruk, Construction and Strength Calculation of space Electrorocket Engine, Moscow, 1970.

[14] M.J.Montazeri, A. Jafar-Gholi, D. Ramesh, J. Abyat, Design of Liquid Propellant Engine, Configuratio and Structure, pp. 329, AIO Publication, 2006 (In Persian).

[15] Ronald W.Humble, Gary N. Henry, Wiley J. Larson , space propulsion analysis and design, United States Department , pp266,1997.

[16] NASA SP-8107, Monograph of National Aeronatics and Space Adminestration, Lewis Research Center, ,Turbopump Systems for Liquid Rocket Engine, NASA Space Vehicle Design Criteria (Chemical Propultion) ,pp.33-34,Agust 1974.

[17] M.J.Montazeri, R. Ebrahimi, New preliminary design method of pump-fed system in $f$ cryogenic lpre base on gradient and genetic algorithms, ModaresMechanical Engineering.Vol.15,No.5,pp.71 80,2015(In Persian).

[18] F-1 ENGINE FAMILIARIZATION TRAINING MANUAL" Section I. R-3896-1, November 1970 